\title{
Das röntgenologisch Erkennbare beim Ulcus duodeni.)
}

Von Dr. Emmo Schlesinger,

Spezialarzt für Magen-Darmkrankheiten in Berlin.

Dem Ulcus duodeni war in den Diskussionen der letzten drei Jahre ein recht breiter Raum zuerteilt gewesen. Wer aber mit Aufmerksamkeit zugehört hat, konnte sich des Eindrucks nicht erwehren, daß das meiste bei ihm noch strittig und im Werden ist. Wenn wir aus seiner Diagnostik den einer objektiven Beurteilung scheinbar am ehesten zugänglichen Teil, den röntgenologischen, herausgreifen, der gleich den anderen eine sehr vielseitige Bearbeitung gefunden hat, so begegnen uns auch hierbei ungemein zahlreiche und weitgehende Widersprüche. Während z.B. der eine Autor [Kreu zfu chs(l)] Hypermotilität als diagnostisch bedeutungsvoll für Ulcus duodeni

1) Vortrag, am 23. II. 1914 d. V. f. Inn. M. u. Kindhlk. in Berlin. (Diskussion S. 1193.) 
anfülırt, nennt der andere [Haudeck (2)] Hypomotilität. In gleicher Weise wird von dell einen Pylorospasmus, von dell anderen Aufhebung des Pylorusreflexes und Pylorusinsuffizienz für Ulcus duodeni bewertet, und ebenso stehen siclı Ptose resp. Hypotonie und Hypertonie, jede die Ulcusdiagnose angeblich belegend, gegenüber. Es darf demnach nicht wundernelimen, wenn Interne und Chirurgen, die derartig widerspruchsvolle Prämissen für ihre Diagnosestellung vorfinden, sich der Röntgenuntersuchung des Ulcus duodeni gegenüber abweisend verhalten und daß man Aussprüchen begegnet, wie sie jüngst noch Sherren (3) getan hat:

„Nach meiner Erfahrung leistet uns die Auskunft, die wir durch die X-Strahlen erhalten, zurzeit keine Hilfe bei der Diagnose. In allen Fällen, bei welchen irgendein $Z$ weifel in dem klinischen Befunde bestand, haben wir von dieser Untersuchungsmethode keinen Nutzen gehabt."

Mit zunehmender Erfahrung habe ich mich immer mehr von dem Werte der Röntgenuntersuchung für das Duodenalulcus überzeugt und glaube, daß an der zeitweilig noch geringen Bewertung derselben zum großen Teil die oben skizzierten Meinungsverschiedenheiten schuld sind, die unausgeglichen in Literatur und Praxis fortbestehell. Ich erachte es deshalb als die dringendste Aufgabe auf diesem Gebiete, zunächst einmal festzustellen, welche Momente es verschulden, daß ernsthafte Autoren in der Beurteilung des gleichen Gegenstandes unter Anwendung der gleichen Mittel zu so ganz verschiedenen Resultaten kommen konnten, und glaube Ihnen nunmehr, gestützt auf ein Material von 114 Fällen, von denen 23 autoptisch bestätigt sind, manche Aufschlüsse hierüber unterbreiten zu können. Was allgemein anerkannt ist und gesichert erscheint, soll nur ganz kurz gestreift werden, um für die Hauptaufgabe Zeit zu gewinnen.

Das Ulcus duodeni ist im Durchschnitt flacher als das Ulcus ventriculi, die Möglichkeit, es unmittelbar röntgenologisch zur Darstellung zu bringen, daher auch seltener gegeben. AuBerdem sind auch die sonstigen mechanischen Vorbedingungen für das Haftenbleiben einer ausreichenden Menge Kontrastbreies im Duodenum sehr ungünstig.

Ich zeige Ihnen hier das größte zur Be-
Fig. 1.

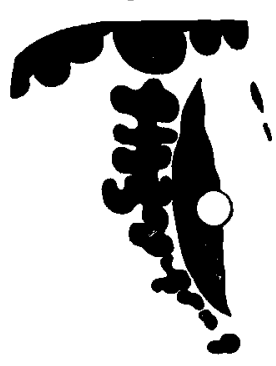
obachtung gekommene Uleus $-7 \mathrm{~cm}$ lang und 2-3 $\mathrm{cm}$ breit - von dem als einzige Zeugen der weitgehenden Zerstörung zwei linsengroße, matte Flecke im Bilde geblieben sind. (Fig. 1.)

Das penetrierende Geschwür kommt am Duodenum absolut und relativ seltener zur Darstellung als am |Magen, aus den gleichen, oben erwähnten mechanischen Ursachen. Ich selbst habe in einem sonst reichlichen Material, in dem sich eine ganze Anzahl von penetrierenden Magenulcera vorfindet, kein gleiches am Duodenum erhalten können und finde auch in der Literatur kaum eins vor, das nicht noch Zweifel an der Richtigkeit seiner Auslegung zuließe. Auch dem schon Geübten droht hier die Gefahr einer Verwechslung, auf die in letzter Zeit mehrfach hingewiesen wurde. [Haudeck (2), Bier (4)], auf die ich aber
Fig. 2.

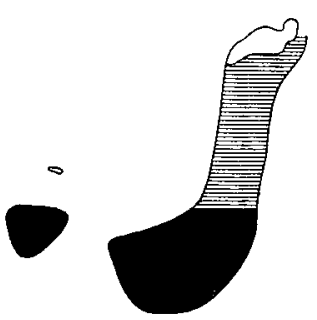

0 aus einem besonderen Grunde noch einmal zurückkomme. Im oberen Duodenalwinkel lagert des öfteren eine kleine Luftblase, die als belanglos in der Regel unbeachtet bleibt. Wenn infolge irgendeines pathologischen Prozesses im oder am Duodenum im Bulbus ein Metallrest zurückgehalten wird, dann kann sich der Inhalt der Pars superior so formieren, da $B$ die drei Schichten schwarz, grau, weiß, genau in der Anordnung wie beim Ulcus penetrans übereinanderliegen und eine verblüffende Aehnlichkeit mit dem radiologischen Bild desselben bekommen. Nun kann sich und hat sich in Wirkliclıkeit mehrfach folgende recht eigentümliche Verwechslung ereignet, die die eigentliche Veranlassung ist, da $\beta$ ich auf diesen Punkt etwas näher eingehe. (Fig. 2.)

Das Röntgenbild zeigt anscheinend die Formation der
Höhle eines Ulcus penetrans oder einer tiefen Nische. Der Befund bei der Operation ergibt eille Höhlenbildung, und dennoclı ist das im Bild Geselıene niclıt die Wiedergabe dieser Nische.

Ich zeige Ihnen hier einen solchen Fall, in dem ein Ulcus penetrans angenommen war. Bei der Operation zeigte sich ein scharf umrandetes, großes Ulcus mit sehr tiefer Nische vor dem Pylorus an der kleinen Kurvatur. Die anfängliche Annahme war, daß die Diagnose im allgemeinen getroffen, nur die Lokalisation nicht präzise war. Bei genauer Ausmessung der Höhlen war es klar, daß der Sachverhalt folgender war: Der Brei war an der Nische vorübergezogen, ohne eine sichtbare Spur zurückzulassen, und hatte sich in dem sonst ganz intakten Duodenum angesammelt.

Das Bild liegt schon längere Zeit zurück. Ist man auf diese Verhältnisse erst einmal aufmerksam geworden, dann ist man vor ähnlichen Irrtümern sicher.

Als anscheinende Seltenheit möchte ich Ihnen folgenden Fall zeigen, den man als Sanduhrduodenum bezeichnen könnte.

Es ist Ihnen bekannt, daß von den Indizien für ein sonst röntgenologisch nicht nachweisbares Ulcus ventriculi eins der verläßlichsten die persistierende Kontraktur der großen Kurvatur im Niveau des an der kleinen Kurvatur liegenden Geschwürs ist, die dem Magen die Sanduhrform gibt. Wieweit in diesem Fall die Einziehung durch einen Spasmus, wieweit durch anatomische Deformierung hervorgebracht ist, muß ich dahingestellt sein lassen, weil die Patientin unoperiert geblieben ist. Die Beständigkeit der Kontraktur geht daraus hervor, daß sie bei der Aufnahme in rechter Seitenlage, die später gemacht ist, ein völlig unverändertes Aussehen zeigt. (Fig. 3a b.)

Von den sekundären Veränderungen des Ulcus beansprucht besondere Beachtung ein Symptom, das unter dem Namen

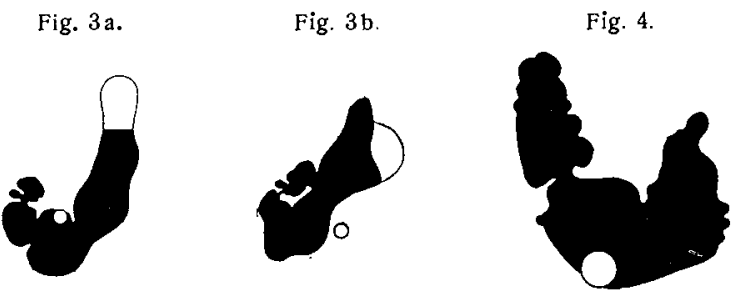

des konstanten Bulbusschattens oder des Dauerbulbus eingeführt ist und das nach Aetiologie und in seiner äußeren Form sehr verschieden sein kann. Einmal kann die Weiterbeförderung des Breies durch Fixierung oder Infiltration der Duodenalwand behindert sein. Hierdurch kommen die verschiedenartigsten Schattengebilde zustande, sehr häufig die von Bier (4) näher beschriebene Zapfenform, bei der nach meiner Erfahrung immer die Wand schwere Veränderungen aufweist. (Fig. 4.)

Häufiger ist aber der Dauerbulbus die Folge einer Dilatation, deren Entstehung dadurch eingeleitet wird, daß beim Ulcus duodeni der Mageninhalt mit erhöhter Wucht und in schnellerer Aufeinanderfolge durch den Pylorus gegen die Duodenalwand geworfen wird. Hierdurch wird diese gedehut, verliert an lokomotorischer Kraft, sodaß der Bulbus noch Reste des letzten Pylorusschusses enthält, wenn der neue ankommt. Wenn die Dilatation erst diesen Grad erreicht hat, ist auch der Circulus vitiosus geschlossen, der zu weiteren erhebliclien Dilatationen führen kann. Man sieht das ursprünglich kleine Gebilde Dimensionen annehmen, die das Mehrfache der anfänglichen Größe ausmachen. Ob das Ulcus selbst retinierend auf den Inhalt wirkt, läßt sich zurzeit nicht sagen; man muß jedoch, so naheliegend diese Annahme auch erscheint, mit der Möglichkeit rechnen, daß ein Ulcus duodeni, ähnlich wie wir das bei Ulzerationen im Kolon durch Stierlin (5) kennen gelernt haben, den Inhalt schnell weiter zu treiben die Neigung hat. Neben allgemeinen Dilatationen finden sich ziemlich häufig am Duodenum isolierte, nischenartige Ausbuchtungen, die aber in seltensten Fällen dem Krater eines Ulcus entsprechen, wie gewöhnlich angenommen wird. Meist waren es durclı irreguläre Adlıäsionen zustande gekommene Deformierungen. Eine seltene Aetiologie hatte ein Fall, bei dem multiple kleine Ulcera, die seit langem abgeheilt waren, so stark gedehnt waren, $\mathrm{daB}$ sie weit über die übrige Duodenalwand linausragten (autoptisch bestätigt). 
Es ist von praktischen Interesse, zu wissen, daß auch mit dieser Möglichkeit zu rechnen ist, weil die Indikationsstellung für die Operation eine wesentlich andere ist als bei Ulcusnischen.

Für die Diagnose der Periduodenitis adhaesiva, der Strikturen und sonstigen deformierenden Prozesse der Duodenalwand gelten die gleichen Ueberlegungen wie an den übrigen Teilen des Intestinaltraktus. Stenosen sind leicht zu diagnostizieren, wenn sie in einiger Entfernung vom Pylorus sitzen.

In folgendem Fall, Fig. 5, ist die Diagnose von der Platte abzu-

Fig. 5. lesen. Bemerkenswert ist, daß es, trotzdem die Stenose Fig. 5. sehr hochgradig ist und das Ulcus 34 Jahre lang bestanden hat, nicht zu der geringsten Erweiterung des Magens gekommen ist, der in sechs Stunden bei kräftiger Peristaltik sich der Kontraktionsmahlzeit von $400 \mathrm{~g}$ entledigt hat, und daß das Ulcus drei Dezennien hindurch eine ausgesprochene Periodizität mit beschwerdefreien Intervallen bis zu drei Jahren Dauer aufwies.

Viel schwerer sind Stenosen dicht hinter dem Pylorus zu diagnostizieren und von der eigentlichen Pylorusstenose zu differenzieren. Das Hauptunterscheidungsmerkmal ist dadurch gegeben, daß bei den im Duodenum liegenden Stenosen schwerere Kompensationsstörungen des Magens entweder garnicht oder erst nach Jahrzehnten auftreten, während sie bei der regelrechten Pylorusstenose schon nach wenigen Jahren vorhanden sind. Im folgenden Fall habe ich allein auf Grund dieser Erwägung die autoptisch bestätigte Diagnose Stenose unmittelbar hinter dem Pylorus gestellt. Die Krankheit hatte 26 Jahre lang gedauert. Der Tonus des Magens ist, wie es das Bild zeigt, trotz erheblicher allgemeiner Vergrößerung, noch recht gut. (Fig. 6.)

Alle bisher demonstrierten Veränderungen im

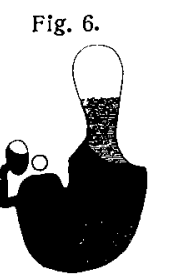
1)uodenum sind anatomischer Natur und vielfach erst nach Jahren soweit ausgebildet, daß sie das Röntgenbild in deutlich erkennbarer Weise verändern. Wäre man auf sie allein angewiesen, dann wäre die Röntgenuntersuchung des Ulcus duodeni eine der undankbarsten Aufgaben. Das Duodenalulcus zeigt abereinen höchst merkwürdigen, in seinem ganzen Umfange sicherlich noch nicht richtig erkannten Einflu $B$ auf den ihm vorgelagerten und mit $\mathrm{ihm}$ in innerstem Konnex und vielseitiger Wechselbeziehung stehenden Magen, dessen Funktionen dadurch in der eingreifendsten Weise verändert werden. Und dadurch, da $b$ gerade das Röntgenlicht uns die Möglichkeit gibt, diese Funktionsänderungen in allen Abstufungen zu verfolgen, werden uns die wichtigsten Indizien für das Vorliegen eines Ulcus duodeni an die Hand gegeben. Aber gerade über diese Magenstörungen, die zunächst rein funkktioneller Natur sind, bestehen die eingangs erwähnten Widersprüche, die für den Fortschritt in der Röntgendiagnostik ein Hemmnis sind. Ich habe nun in mehrjährigen Beobachtungen den bestimmten Eindruck gewonnen, daß die Beeinflussung der Magenfunktionen durch das Ulcus duodeni keine wechselvolle, wie von den meisten bisher angenommen wird, und keine widerspruchsvolle, sondern eine sich stets gleichbleibende ist, und zwar stets im Sinne einer Steigerung der Funktionen, d. h. anscheinend sämtlicher Funktionen, sich geltend macht, und daß die vorhandenen Widersprüche unter konsequenter Einhaltung dieses Gesichtspunktes sich restlos oder nahezu restlos lösen lassen. Die Bestätigung der Annahme einer stets gleichsinnigen Einwirkung des Ulcus duodeni auf die Magenfunktionen wird in Zukunft bei der Mehrzahl der Duodenalulcera entscheidend sein für den Wert der Röntgenuntersuchung. Ich muß deshalb bei diesem Punkt bei den Teilerscheinungen der sogenannten Magenneurose etwas länger verweilen.

Am meisten in die Augen fallend ist die Störung der Peristaltik. In reinen Fällen ist die peristaltische Bewegung nach jeder Richtungsmöglichkeit gesteigert: Tiefe, Geschwindigkeit, zeitlicher und örtlicher Beginn. Dies-ist vielfach beobachtet und als Teilerscheinung einer Reflexwirkung des Ulcus auf den Magen zugegeben. Nur sind eine Reihe von Ausnahmen aufgestellt, bei denen die Reflexsteigerung angeblich ausbleibt oder sogar eine Reflexlähmung vorliegen soll. Und hiergegen möchte ich mich wenden. Richtig ist, daß gelegentlich die
Peristaltik schwach erscheint oder iiberhanpt nicht sichtbar ist, richtig ist aber nicht, daß dies die Folge einer Reflexlähmung ist. Vielmehr liegen hier ganz andere, außerhalb des unmittel. baren Wirkungsbereiches des Ulcus duodeni befindliche Ursachen vor. Hierher gehören Beobachtungen, wie ich sie wiederholt in den letzten Jahren gemacht und in einer neueren Arbeit über die sekretorischen Vorgänge im Magen zusammengestellt habe. Man sieht bisweilen - nach Verabreichung einer Kontrastmahlzeit - an Stelle des gewöhnlichen Füllungsbildes einen zentral gelegenen, kugeligen, dunklen Schatten, umrahmt von einem bis zu mehreren Zentimetern breiten, hellen Streifen in folgender Anordnung. (Fig. $7 \mathrm{a} \mathrm{b.)}$

Bei diesem Füllungsbild ist die Peristaltik, gleichviel welches Grundleiden vorliegt, schwach. Zweifellos war der dunkle Schatten der Kontrastbrei, der helle Streifen Sekret. Trat bei weiterer Füllung eine gute Schichtung des Inhaltes ein (Fig. 7 b), dann waren auch Wellen in der zu erwarten. den Stärke sichtbar. Die zuerst auftauchende Vermutung, daß die Mattheit des Schattens das Erkennen der peristaltischen Bewegung

erschwerte, diese selbst @aber vorhanden war, mußte fallen gelassen werden, weil auch bei genauestem Zusehen unter starker Abblendung, bei dem die Konturen der Magenwand deutlich sichtbar waren, die Peristaltik unverändert schwach erschien.

Ich nehme deshalb an, daß die der Magenwand anliegende Sekretschicht es verhindert, daß der peristaltische Impuls sich in der Bildung großer Wellen äußern kann und daß hierzu der taktile Reiz einer konsistenteren Nahrung erforderlich ist. Bestätigt finde ich diese Annahme in Beobachtungen von Flesch und Peteri (6), daß der Säuglingsmagen bei reiner Milchkost keine Peristole und keine radiologisch wahrnehmbare Peristaltik zeigt, diese dagegen bei festerer Nahrung sofort sichtbar werden. Die Rolle, die hier die Milch spielt, hatte in meinen Fällen die der Magenwand anliegende Sekretzone. Westphal und Katsch (7) berichten über Fälle von Ulcus duodeni, in denen der Magen prall mit Sekret gefüllt war und ebenfalls keine Peristaltik zeigte und die sie als maximalsekretorische den hyperperistaltischen gegenüberstellen.

Eine zweite Gruppe von Duodenalulcera, bei denen man die Hyperperistaltik vermissen kann, ohne daß deshalb die Reflexsteigerung fehlt, ist die mit Gastroptose plus Dilatation komplizierte. Wenn die ptotische Dehnung hochgradig ist, die Muskeln der Pars media sehr viel von ihrer Kontraktilität verloren haben, dann kann der vom Ulcus ausgehende Impuls zur Hyperperistaltik dort auch gelegentlich frustral verlaufen. Das Gleiche kann bei den Dilatationen und Ektasien vor den Stenosen geschehen. Hier treten während der stark verlängerten Verweildauer der Speisen in der Regel Ermüdungszustände der Magenmuskulatur von bisweilen stundenlanger Dauer auf, in denen die Peristaltik vollständig ruht. Auch hier muß sich schließlich der reflektorische Impuls erschöpfen. Bei den beiden letztgenannten Gruppen ließ sich der scheinbar erloschene Rcflex in folgender Weise wieder sichtbar machen. Es ist bekannt, daß durch meclianische Irritationen der Magenwand, wie wir sie durch plötzliche scharfe Kontraktionen der Bauchdecken besonders bequem erreichen, dje Peristaltik angeregt wird. Wenn man die gleiche Prozedur nun bei einem Magen, der unter einem momentan latenten Reflex steht, vornehmen läßt, sieht man bisweilen eine ganz enorme Steigerung der Peristaltik akut einsetzen. Die eben noch glatten Wandungen des völlig ruhenden Magens sind zu hohen Wellen aufgeworfen, die in starker Erregung zum Pylorus eilen. Die Hyperperistaltik ist eine sehr hartnäckige Begleiterscheinung des Ulcus duodeni. Ich habe sie wiederholt bei Kontrolluntersuchungen nach einer mit vollem Erfolge durchgeführten Behandlung bestehen sehen und glaube, daß sie Monate, vielleicht Jahre hindurch, wenn auch in wechselnder Stärke, besteht. Es ergibt sich also: Die in der Hauptsache die Peristaltik besorgende Ringmuskulatur befindet sich beim Ulcus duodeni lange Zeit hindurch im Zustande erhöhter reflektorischer Erregbarkeit. In den Fällen, in denen die Peristaltik vermißt wurde und man eine verminderte Erregbarkeit annehmen zu können glaubtc 
und die man als Gegensätze den ersteren gegenüberstellte, liegt nicht ein Ausbleiben der Reflexerhöhung vor, sondern nur ein Niederhalten seiner sichtbaren Aeußerung durch fremde Kräfte. In dem Augenblick, in dem diese zurücktreten, wird die peristaltische Reflexsteigerung wieder manifest.

Ein zweites Symptom der Magenneurose ist die Hypertonie, die sich in verstärkter Peristole und in bestimmten Formveränderungen kundgibt. Der Begriff der Hypertonie existierte aber schon in der Röntgenliteratur, bevor viel von einer Reflexwirkung des Ulcus duodeni auf den Magen bekannt war. Sie ist das Charakteristikum des sogenannten Stierliornmagens und sollte sagen, daß dieser Stierhornform eine das normale Durchschnittsmaß überschreitende Muskelkraft innewolnt. Er bezeiclınet den höchsten seinerzeit bekannten Grad von Tonus dicht an der Grenze des Pathologischen. Die beiden in ihrem Wesen ganz verschiedenen Begriffe von Hypertonie werden aber leider unterschiedslos nebeneinander gebraucht, und es wird dem Leser überlassen, jedesmal den richtigen zu finden. Der große Unterschied im Zustande der beiden Arten von Hypertonie markiert sich am deutlichsten im Bilde. (Fig. 8.)

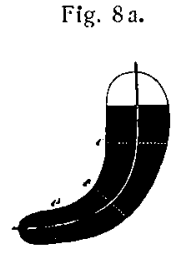

1. Lage diagonal.

2. Kurvaturen nach oben divergierend.

3. Schwebend bei ausgeglichenem abdominalen Druck.

4. Die Achse gleichmäBig leich gekrümmt zum Pylorus ziehend.
Fig. $8 \mathrm{~b}$.

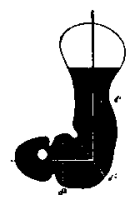

Steil.

$$
\text { Parallel. }
$$

Gestreckt, mit erhötem Druck die Nachbarorgane beiseite schiebend. Rechtwinklig geknickt in dem unteren Drittel, sodaß eine reguläre Pars horiontalis entsteht.
Die Unterschiede der beiden Formen sind so markant, daß sie nicht gesucht zu werden brauchen. Die charakteristische Form beim Ulcus duodeni wird hervorgebracht dadurch, daß die Längsmuskulatur in den Zustand einer Kontraktur versetzt wird, in dem sie verharrt, während der Magen seine sonstige Tätigkeit weiter ausübt, die sie wohl mehr oder weniger alteriert, aber nicht inhibiert. Der Kontraktionszustand ist reflektorisch, und es empfiehlt sich deshalb, diese Hypertonie, um einer Verwechslung mit der ersteren zu entgehen, als reflektorische $\mathrm{zu}$ bezeichnen. Beiden Mägen gemeinsam ist, daß sie zunächst in der Regel klein sind. Ein Unterschied liegt darin, daß der Stierhornmagen seine Größe andauernd unverändert beibehält, während der reflektorische infolge meist am Pylorus einsetzender Spasmen, über die gleich eingehender gesprochen werden soll, den Keim zu kranklıafter Erweiterung in sich trägt. Der Einflu $B$ der Hypertonie auf die sonstige Tätig. keit des Magens ist noch wenig studiert. Das Auffallendste ist, $\mathrm{da} ß$ der Magen starr und steil gestellt aussieht. Die Hypertonie erlıölıt den Innendruck des Magens und gibt Anlaß zu schmerzhaften Sensationen. Die Starrheit der Längsmuskulatur muß aber auch für die fortschreitenden Kontraktionen der Ring. muskulatur, d. h. für die Peristaltik, ein Hemmnis sein. Sicher ist, daB es diesem Umstande zuzuschreiben ist, wenn bei hochgehenden, sichtlich erregten Wellen und gleichzeitig bestehender Hypertonie die sonst so schnell eilenden Wellen auffallend lang. sam sich bewegen und bisweilen $\mathrm{zu}$,,stehenden" Wellen werden. In gleicher Weise wie bei der Peristaltik stehen im vollen Gegensatz zu dem meist erhobenen Befund der Hypertonie die Angaben einiger Autoren, daß sie Hypotonie resp. Ptose in der Regel als Begleiterscheinung des Ulcus duodeni gefunden haben und daß deshalb Tonusgrade für ein Ulcus duodeni überhaupt nicht zu verwerten sind. Ganz analog wie bei der Peristaltik sind auch hier diese Widersprüche zu erklären. Einmal kann das Ulcus duodeni, wenn auch anscheinend viel seltener, bei einem vorher ptotischen Magen entstehen. Trifft der Duodenalreflex einen solchen Magen, dann kann er die Hypertonie und Ptose nicht aufheben; er kann wohl bewirken, daß die tiefstehende Kurvatur etwas ansteigt, daß die Peristole etwas kräftiger wird, er kann aber den tiefhängenden, schlaffen Magen niemals zu den oben skizzierten hypertonischen redressieren. Aehnliches wie die Ptose kann die zn kktasie himneigende allgemeine Erweiterung bewirken. In den gedelinten Wandungen hat sich der Duodenalreflex erschöpft. Mall merkt ihnen zeitweise nichts von der ihnen innewohnenden Exzitation an. Vgl. Fig. $7 \mathrm{~b}$.

Magen mit grober Motilitätsstörung (11 Stunden Verweildauer). Und wieder analog den Vorgängen bei der Hyperperistaltik führen wir den Magen etwas brüske äußere Impulse zu, und im Moment sehen wir ihn sich aufbäumen, stärker und steiler werden, die große Kurvatur wesentlich höher steigen, kurzum eine deutliche Hypertonie dokumentieren (Fig. 9). Wenn aber die plötzliche Steigerung der Peristaltik auch bei ganz normalen Mägen als etwas ganz Gewöhn. liches vorkommt und nur das Exzessive dabei krankhaft ist, sieht man deutliche Tonusänderungen bei normalen viel seltener. Ihre Konstatierung ist ein nicht $\mathrm{zu}$ vernachlässigender Beitrag für die Diagnose. Für die Dauer der Hypertonie gilt das Gleiche

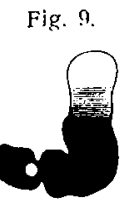
wie für die Peristaltik, da sie ja gleichen Einflüssen entspringen. Solange das Ulcus besteht, hält es den Magen unter tonussteigerndem Reflex. Wie lange noch darüber hinaus der Reflex besteht, muß erst jahrelange Beobachtung ergeben. Sicher ist, daß er auch in recht hoher Intensität nach Umwandlung des Ulcus in eine feste Narbe angetroffen wird. Die widerspreclienden Angaben einiger Autoren sind auch darauf zurückzuführen, daß der durch Gegenkräfte wie Dilatation und Ektasie niedergehaltene Reflex nicht erkannt und es nicht versucht wurde, ihn künstlich zu erwecken. Die Wirkung des Duodenalulcus auf den Magentonus ist stets steigernd, niemals depressiv.

Auf die $\mathrm{Hypersekretion} \mathrm{möchte} \mathrm{ich} \mathrm{hier} \mathrm{nicht} \mathrm{näher}$ eingehen, da wir doch in der Regel die Sonde einführen und die röntgenologische Sekretionsprüfung ein sehr umfangreiches Kapitel für sich darstellt. Nur soviel sei erwähnt, daß nach meiner Erfahrung das Duodenalulcus die Magensaftsekretion zunächst stets steigert, daß aber auch hier sekundär schließlich Erschöpfungszustände und gastritische Veränderungen zu Sekretionsverminderungen führen können, sodaß ab und zu selbst Achlorhydrie angetroffen wird. Ob ein Ulcus bei Achlorhydrie noch entsteht, ist, soweit mir die Literatur bekannt ist, bisher nicht erwiesen.

Von weitergehendem Interesse sind zwei andere Begleiterscheinungen der Magenneurose: der Pylorospasmus und die Pylorusinsuffizienz. Sie sind die Extreme der pylorischen Zustandsmöglichkeiten, und trotzdem sind sie beide als für Ulcus duodeni charakteristisch in Anspruch genommen worden, also der gleiche Widerspruch, der sich auch bei den anderen Aeußerungen der Magenneurose vorfindet. Wenn ich im Folgenden auch hier eine Lösung versuche und glaube, daß sie gelungen ist, so möchte ich die Theorie des Pawlowschen Säurereflexes zunächst ganz aus der Erörterung lassen und möchte mich nur an wirklich Beobachtetes halten. Zum Ausgangspunkt nehmen wir den reflektorisch-hypertonischen Magen mit Hyperperistaltik, wie ihn Fig. $8 \mathrm{~b}$ wiedergibt. Wenn man hiervon eine größere Anzahl beobachtet, fällt bei gleich hoch einsetzenden und gleich tiefen Wellen auf, daß ihre Geschwindigkeit ganz verschieden ist. Bisweilen eilen sie rapide zum Pylorus, bringen schnell das Antrum zur Kontraktion, sprengen mit leichter Mühe die pylorische Kontraktion und schleudern einen Bissen aus dem Magen heraus, der dann durch das Duodenum geradezu hindurchfliegt: Hypermotilität, wie sie ungefähr zuerst von Barclay (8) gezeichnet wurde. Schon hier setzen die Differenzen ein. Viele erblicken bei dieser Art von Hypermotilität eine Aufhebung des duodenalen Pylorusreflexes: Pylorusinsuffizienz. Ich sehe keinen Grund, zur Erklärung dieses raschen Durcheilens durch das Duodenum das Bestehen einer Pylorusinsuffizienz als Folge der Aufhebung des normalen Pylorusreflexes anzunehmen. Die Hyperperistaltik allein genügt zur Erklärung dieser Art von Hypermotilität, eine Hyperperistaltik, der am Pylorus kein besonderes Hindernis erwächst. Auch Westphal und Katsch (7) und ebenso Huerter (13) sind gegen die Annahme einer Insuffizienz des Pylorus. Neben dieser Hypermotilität findet 
sich beim Ulcus duodeni häufig Hypomotilität, eine VerlängeI'ung der Austreibungszeit, und diese wird bei anatomisch freiem Pylorus in der Regel auf einen Spasmus am Pylorus zurückgeführt. Mit dem Worte Spasmus ist aber der Zustand des Pylorus, der die zunächst beim Ulcus duodeni bestehende Hypermotilität aufhebt, nicht ganz treffend bezeichnet. Was man im Bilde beobachtet, ist Folgendes: Die oben beschriebene große Geschwindigkeit der Wellen ist gewichen. Sie ist normal oder auch weit unter normal geworden. Vielfach sielit man tiefeinschneidende Wellen völlig stillstehen, vielfach ziehen sie ruckweise, aber im ganzen doch zögernd zum Pylorus hin. Langsam und schwer kontrahiert sich das Antrum und aucli der Pylorus. Der schattenfreie Raum, der den Kontraktionsring kennzeichnet, wird viel breiter, $3-4$ mal so breit als normal, und kann auch 3-4 mal solange bestehen bleiben. Man sieht, welche Anstrengung und Zeit es kostet, diesen festen und breiten Verschluß wieder zur Lösung zu bringen, und kann diese Erschwerung der peristaltischen Tätigkeit, die sich für den Träger des Leidens meist völlig unbemerkt vollzieht, während der Spasmus häufig vehemente Schmerzen, „Krämpfe“, auslöst, während eines großen Teiles der Magenverdauung verfolgen. Dieser Zustand ist zweifellos gleichfalls eine Hypertonie, eine Teilerscheinung der gesamten Magenhypertonie. Von dem gemeinhin gebrauchten Begriff Spasmus unterscheidet sie sich sehr wesentlich. Der Spasmus scliafft einen VerschluB, der nicht mehr, wenn auch nur zeitweise, überwunden wird. Die Hypertonie des Pylorus erschwert und verzögert nur die Oeffnung, hindert sie aber nicht. Beide, Pylorushypertonie und Pylorospasnus, schaffen Motilitätsstörungen, der Spasmus im allgemeinen die gröberen. Für die Diagnose Pylorospasmus ist der Nachweis erforderlich, daß der Pylorus wirklich geschlossen bleibt und Mageninhalt ihn nicht passieren kann. Vielfach ist aber die Röntgendiagnose Pylorospasmus eine Phantasiediagnose, die sich ausschließlich auf die festgestellte Motilitätsstörung stützt. Es wird sogar gelehrt: Wenn bei anatomisch freiem Pylorus eine Verweildauer von mehr als 6 bis höchstens 8 Stunden gefunden wird, so bedeutet das Pylorospasmus. Dieser Satz ist sicher für viele Fälle unzutreffend. Narben, Adhäsionen. Ulcera, Tumoren am Corpus ventriculi, Lageanomalien und Dilatationen am Magen und Duodenum, psychische Depressionen akuter und protrahierter Art, akute Gastritiden und zahlreiche andere Momente können auch ohne jeden Spasmus eine Verweildauer der Speisen weit über 8 Stunden hinaus verscliulden. Das viel mißbrauchte Wort Pylorospasmus sollte mit viel größerer Vorsicht als bisher gebraucht und muß auch gegen die Hypertonie des Pylorus sorgfältig abgegrenzt werden.

Für das Bestehen einer Pylorusinsuffizienz ist ferner seit Jaliren bis in unsere Tage geltend gemacht worden, daß sofort nach dem ersten Bissen ein Uebertritt in das Duodenum erfolgt. Doch erübrigt sich auch hier die Annahme einer besonderen Pylorusinsuffizienz, zur Erklärung für das vorzeitige Erscheinen des Duodenalscliattens genügt ebenso wie für das rasche Durcheilen die Hyperperistaltik. Daß in Wirklichkeit auch der Reflex hier voll erhalten ist, geht aus den präzisen scharfen Pyloruskontraktionen hervor, die hierbei gut $\mathrm{zu}$ beobachten sind. Niclit zu verwechseln mit dieser angeblichen Pylorusinsuffizienz ist ein Offenstehen des Pylorus, das sich beim Ulcus duodeni aus zweifacher Ursache vorfindet. Einmal die anatomische, infolge von Infiltrationen, Adhäsionen und sonstigen Wandveränderungen. Ferner aber, und dies dürfte am häufigsten mit einer Reflexhemmung verwechselt werden, können die Längsfasern, soweit sie am Pylorus enden, bei ihrer Kontraktion den Pylorus aufreißen und offenhalten. Es ist also dieses Offenstehen wiederum die Folge einer Reflexsteigerung, die sich an den Längsmuskeln vollzieht und am Pylorus äußert. Es sprechen also sämtliche bisher aufgeführten Vorgänge am Pylorus ebenfalls für eille in ganz einheitlichein Sinne sicl vollziehende, vom Duodenalulcus ausgehende Reflexsteigerung.

Wie bei allen Hohlorganen, treten aucli im Magen sehr bald als Folge der funktionellen Anomalien anatomisclie Veränderungen auf, die siclı in isolierten oder allgemeinen Hypertrophien und Erweiterungen äußern. Und gerade diese sind es, die dem Magenbilde beim Ulcus duodeni sein besonderes Gepräge geben. Die Trennung der primär funktionellen von den sekundär anatomischen Veränderungen, die bisher meines Wissens niemals ganz durchgeführt wurde, ist für die bisweilen sehr schwierige Differentialdiagnose von großer Bedeutung. An erster Stelle möchte ich ein viel diskutiertes Symptom, dic hohe Rechtslage des Pylorus, erörtern. Im allgemeinen wird gesagt, da $\beta$ sie durch Traktion infolge periduodenitischer Narbenschrumpfung entsteht. (Fig. 10.)

Mir war aber aufgefallen, daß selır häufig, auch wenn siclı weder röntgenologisch noch sonst klinisch Adhäsionen naclweisen ließen. der Pylorus ganz auffällig weit rechts und hocli lag. Es war schwer, dies zu erklären, wenn Konstitution des Kranken, Magerkeit und Flachheit des Abdomens einen ortho-

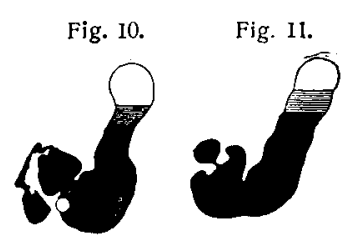

$\circ$ resp. hypotonischen Magen mit viel tiefer und viel weiter links liegendem Pylorus erwarten ließeIs. Gehäufte Beobachtungen der folgenden Art schienen mir die Frage völlig zufriedenstellend $\mathrm{zu}$ beantworten. Außer den beiden oben skizzierten Typen peristaltischer Abnormitäten, der beschleunigten und vertieften, die scheinbar ungehindert den Mageninhalt weiterbefördert, und der verlangsamten und vertieften, die an der Hypertonie des Pylorus ein Hemmnis findet, sieht man noch eine dritte Kombination: beschleunigte und vertiefte Peristaltik plus ausgesprocliener Hypertonie des Pylorus. (Fig. 11.)

Hier eilen tiefe, energische Wellen zum Pylorus, der' siclı im Zustande übernormaler Anspannung und Kontraktur befindet. Die Welle ist nicht imstande, den Verschlu $B$ sofort zu öffnen. Sie staut sich vor ihm wie an einem Wellenbreclier, schlägt über ihn hinweg und reißt ihn ein Stück mit sich fort. Die Strömung hat sich noch niclit ausgeglichen, da kommt schon eine neue Welle lieran, die wieder über den Pylorus hinüberschlägt, sich mit der ersteren vereint und zu weiter Dehnung der Magenwand führt. Und so fort, Welle für Welle. Dem Auge erscheint es, als ob ein starker Kraftaufwand nötig ist, um diese breite Expansion der Magenwand zu Wege zu bringen, die den Pylorus vor sich liertreibt und ilın dann noclı nach sich zieht. Bei jeder Nahrungsaufnahine und bisweilen während der ganzen Dauer ihrer Eliminierung aus dem Magen kann sich das gleiche Spiel wiederholen. Die Richtung, in der die Bewegung sich vollzielit, ist vorgeschrieben. Sie gelit gegen den Scheitel des Duodenums hill, also nach rechts oben. Es handelt sich demnach in den Fällen von hoher Reclitslage ohne Adhäsion in Wirklichkeit um eine Propulsion des Pylorus und nicht um eine Traktion. Wenn sich diese Annahme bestätigt, dann wäre auch das oft beschriebene Bild der hohen Rechtslage plus Adhäsion für viele Fälle anders als bisher zu deuten. Nicht anfängliche Verwachsung und nachherige Schrumpfung zieht den Pylorus nach rechts oben, sondern Hyperperistaltik bei hypertonischem Pylorus drängt ihn nach reclits oben, wo ihn dann sekundäre Verwachsungen fixieren. Eine kleine Verlagerung des Pylorus kann die Hypertonie allein zuwegebringen, das erwähnen schon Baron und Barsony (9). Die holı Rechtslage des Pylorus bestelıt in Fällen ohne Adhäsionen voraussichtlich nur während der Verdauungsperiode, wenn die Peristaltik im Gange ist, in der Ruhepause sinkt der Pylorus zugleich mit der Relaxation des Magens sicherliclı nach links und unten zurück.

Die Propulsion des Pylorus gegen das Duodenum lin hat häufig eine Sclırumpfung der Pars superior duodeni, wie sie auch in obigem Bilde (Fig. 10) zum Ausdruck kommt, zur Folge. Als weitere Veränderungen, die auf eine besonders lange Dauer und selur liohe Intensität der primär funktionellen Symptonte hinweisen, die aber olme weiteres verständlich sind und bei denen ich mich deslialb nicht länger aufhalten möchte, seien erwähnt eine isolierte Dilatation der Pars pylorica, die sich in die Konkavität des Duodenums einbettet und diese scliließlich naclı rechts hin überlagern kann; ferner eine allgemeine Verbreiterung und schließlich auclı Verläıgerung des Magens. Diese sekuıldäreı Veränderungen einscliließ. 
lich der Propulsion des Pylorus sind im einzelnen Falle von größerer Bedeutung für die Ulcusdiagnose als die primär funktionellen Störungen.

Wenn wir diesen ganzen vielgestaltigen Komplex der Magenneurose kurz cliarakterisieren wollen, so müssen wir uns vergegenwärtigen, daß die primären Veränderungen sämtlich einen Erregungszustand, eine Exzitation kennzeiclınen. Wir dürfen darum den Zustand, in dem der Magen sich beim Ulcus duodeni befindet, zwanglos als Exzitationsneurose bezeichnen. Die Bezeichnung hat auch für die späteren Stadien des Ulcus duodeni Geltung, wenn Ermüdungs- und Erschlaffungszustände das Bild komplizieren und anatomische Veränderungen hinzutreten. Aus dieser Auffassung heraus erscheint das Gesamtbild des Ulcus duodeni, gleichviel, ob wir es klinisch oder röntgenologisch allein betrachten, ungleich einleitlicher und auch in seinem subjektiven Teil und sensorischem Verhalten viel verständlicher. Es verliert dann viel' von der Buntheit und Verworrenheit, die ilım so oft zum Vorwurf gemacht wird. Diese Auffassung ist entstanden unter Beachtung und Weiterentwicklung von Theorien aus der jüngsten Zeit über den Zusammenhang zwischen Ulcus pepticum und Magenneurose, an deren Ausarbeitung besonders Lichtenbelt (10), Rössle (11), v. Bergmann (12), dessen Assistenten Westphal und Katsch (7) u. a. m. beteiligt sind. Die Ansichten der einzelnen Autoren zeigen Differenzen, deren Detaillierung den Rahmen dieses Themas überschreiten würde. Nur auf das Klinische, soweit es sich im Röntgenbilde wiederspiegelt, müssen wir etwas eingehen. In seiner jüngsten Publikation faßt v. Bergmann (12) das Resultat seiner bezüglichen Arbeiten dahin zusammen, daß das Ulcus duodeni ebenso wie das Ulcus ventriculi meist mit Zeichen gestörter Innervation am Magen einhergeht und daß ebenso in einer großen Mehrzahl der Fälle ein geändertes Verhalten des vegetativen Nervensystems an anderen Organen nacliweisbar ist. Es ist zweifellos das Verdienst v. Bergmanns, zuerst nit besonderen Nachdruck auf das Vorliegen dieser Magenneurose hingewiesen zu haben, die Westphal und Katsch treffend als ,krankhafte Tätigkeitssteigerung" bezeichnen, die in allen ihren Fällen nachweisbar war. Sie sind also bezüglich des Befundes zu einer prinzipiell gleichartigen Auffassung über die Vorgänge am Magen gelangt, die icl als Exzitationsneurose bezeichnen möchte und bei der ich das Zusammenwirken und Gegeneinanderwirken der einzelnen Kräfte oben ausführlich dargelegt und den Nachweis zu führen versucht habe, daß auch da, wo die Ueberfunktion in das Gegenteil scheinbar verwandelt ist, der exzitierende Reflex latent fortbestelit, und daß sich in dieser Weise die zahlreichen der Einheitlichkeit in der Auffassung der neurotischen Erscheinungen entgegenstelıenden Angaben widerlegen lassen. Mit Westphal und Hetsch möchte ich glauben, daß der Dauerbulbus und die Ektasie neurogen erklärt werden können, und als Novum, abgesehen von einigen oben geschilderten Dingen, noch die Propulsion des Pylorus während der Digestion hinzufügen. So vielfältig sich demnach rein symptomatisch die Auffassung v. Bergmanns und seiner Schüler mit der von mir soeben dargelegten deckt, so gehen sie bezüglich der Aetiologie auseinander. v. Bergmann faßt die Magenneurose als Teilerscheinung einer erhöhten Reflexerregbarkeit auch des weiteren Nervensystems, besonders des Vagus, auf und sieht in dem Ulcus meistens die Folge einer spastischen Ischämie der Schleimhäute, die den Magenfermenten Gelegenheit zum Angriff auf die Substanz gibt. Ich möchte zunächst noch annehmen, daß der ganze umfangreiche, oben geschilderte Symptomenkomplex der Magenneurose reflektorisch von dem Ulcus ausgelöst wird, und deshalb ganz vollständig den Zustand des Magens als reflektorische Exzitationsneurose beim Ulcus duodeni bezeichnen, die auch neben einem sonst intakten Nervensystem bestehen kann. Weitere Studien müssen ent. scheiden, welche Auffassung zu Recht bestelit, oder ob sie beide partiale Geltung haben und jede nur auf bestimmte Gruppen zutrifft.

Und nun zur Differentialdiagnose, dem schwierigsten Punkt. Was bisher auch von den besten Freunden der Röntgenuntersuchung - so noch ganz kürzlich von Bier - behauptet wurde, kann ich auch nach erneuter Sichtung der Symptomatologie bestätigen: Keines der zahlreichen Symptome ist für sich allein entscheidend; dennoch habe ich gefunden, daß von den drei Hauptstützen der Diagnose: Anamnese, okkulte Blutungen, Röntgenbild, das letztere öfter die Entscheidung bringt, nachdem die beiden ersteren versagt liabell. Icl möchte ganz kurz die häufigsten Affektionen, mit denen das Duodenalgescliwür in der Diagnose konkurriert, durchgelien:

1. Die Neurasthenie, besonders die mit Hyperchlorhydrie, kann im Status und in der Anamnese ein dem Ulcus duodeni selır ähnliches Bild geben., Im Röntgenbilde sieht man wohl vielfach die Form der reflektorischen Hypertonie oder Hperperistaltik, dagegen fehlen die ausgeprägten Sekundärerscheinungen. Ich zeige Ihnen hier einen Fall von Hypertonie (Fig. 8b) und ferner eine Hyperperistaltik (Fig. 12), die mir beide mit der Wahrscheinlichkeitsdiagnose Ulcus duodeni zugeschickt waren.

Ulcus duodeni war durch die Röntgenuntersucliung unwahrscheinlich gemacht, und im weiteren Verlauf konnte man ein solches auch mit Sicherheit ausschließen. Eine ernste Gefahr zur Verwechslung hat in den zur Beobachtung gekommenen Fällen nicht bestanden.

2. Die Cholelithiasis macht schwere Erscheinungen am Magen nur ins akuten Anfall. Ich hatte Gelegenheit, einen Fall zu beobachten, in dem bei der ersten Durchleuchtung der Magen absolut normal nach Form und Funktion sich erwies. Nach einer Stunde trat ein vehementer Kolikanfall ein und mit ihm eine intensive allgemeine Kontraktur der Magenmuskulatur. Von einer nennenswerten reflektorischen Beeinflussung im latenten Stadium war hier nichts zu sehen, und sie ist auch anderweitig nicht beobachtet worden. Aehnliches gilt für die Nephrolithiasis, Pancreatitis und analoge Erkrankungen; aus diesem Grunde fehlen auch hier die sekundären Veränderungen.

3. Die clironische Appendicitis könnte der Diagnose schon gefährlich werden, weil von ihr andauernd exzitierende Reflexe nach allen Teilen des Abdomens und auch zum Magen ausgehen. Doch sind auch lier die Reflexe wesentlich schwäclier als beim Duodenalulcus, und ich kenne zurzeit keinen Fall, der $\mathrm{zu}$ so weitgehenden anatomisclıen Folgezuständen geführt hätte, wie wir sie beim Ulcus duodeni meistens sehen. Das Gleiche gilt von Genitalerkrankungen, Adhäsionen im Abdomen und ähnlichen Affektionen. Was das Ulcus duodeni eben "vor allen anderen Affektionen in seiner Wirkung auf"den Magen auszeiclinet, ist die Kontinuierlichkeit plus Intensität, die es dadurch erhält, daß es in inniger funktioneller Gemeinschaft mit dem Magen sich befindet, den es mit seinen Reflexen vollkommen zu beherrschen scheint. Die Kontinuierlichkeit der Beeinflussung möchte iclı neben der Intensität noch besonders hervorheben. Mehrmals habe ich Folgendes beobachten können: Die durch Anamnese, dauernde Blutungen und charakteristisches Röntgenbild sichergestellte Diagnose gab Veranlassung zu einer typischen Ulcuskur in der Klinik. Vier Wochen später Blutungen beseitigt, sämtliche Beschwerden beseitigt, starke Gewichtszunahme, klinisch anscheinend völlige Heilung. Die Röntgenuntersuchung ergibt aber nahezu unverändertes Bestehen der Exzitation des Magens. Drei Monate später bei völligem Woblbefinden fast der gleiche Röntgenbefund. Diese stark protrahierte Einwirkung, die anscheinend von der Narbe zunächst wenigstens die gleichen Impulse empfängt wie von dem offenen Ulcus, scheint mir am ehesten die Möglichkeit zu geben, andere Affektionen auszuschalten. Sehr große Schwierigkeiten bereiten zwei Affektionen:

4. Das Ulcus pylori. Die Anamnese kann völlig der des Ulcus duodeni gleichen, ebenso der gesamte klinische Symptomenkomplex. Nach zahlreichen eingehenden, die Differentialdiagnose von Anfang an berücksichtigenden Untersuchungen, zu denen auch die Kinematographie herangezogen wurde, erblicke ich ein Unterscheidungsmerkmal im wesentlichen nur darin, daß das Ulcus pylori mehr echte Spasnien, das Ulcus duodeni mehr hypertonische Zustände am Pylorus 
auslöst und daß deshalb die Erscheinungen der echten Pylorusstenose, Ektasie, Rechtsdistanz im Straussschen Sinne beim Ulcus pylori viel früher auftreten können und schon nach wenigen Jahren einen Grad erreichen, wie er beim Ulcus duodeni auch nach Jahrzehnten nicht beobachtet wird. (Vgl. auch Fig. 6.) - Und ferner

5. die chronische Cholecystitis mit Adhäsionen. Bei ihr wie beim Ulcus duodeni findet sich hohe Rechtslage des Pylorus. Außerdem gehen die Adhäsionen von der Gallenblase meist zum Duodenum oder Pylorus hin, auch gehen meist starke Reflexe zum Magen. Hier hat die Röntgenuntersuchung öfters völlig versagt, nicht ein Symptom fand sich vor, auf das man sich hätte verlassen können. Die anatomischen Sekundärerscheinungen waren bei der Cholecystitis adhaesiva chronica ähnlicher Art, wie wir sie auch beim Ulcus duodeni antreffen.

Die bisherigen Ausführungen stützen sich auf ein Material von 114 Fällen von Ulcus duodeni, von denen 23 operiert wurden und die einem Zugangsmaterial von ungefähr 5000 Magendarmfällen in 2-3 Jahren entstammen. Eine Nachprüfung des gesamten Materials hat aber ergeben, da $B$ noch vor $1 \frac{1 / 2}{2-2}$ Jahren mancher Fall unter der Diagnose Gastritis acida oder Hyperazidität ging, der sich später als sicheres Ulcus erwies. Wie oft dies heut noch geschieht oder auch das Umgekehrte, daß ein Fall als Ulcus imponiert, der keines ist, muß die Zukunft lehren. $\mathrm{Zu}$ bemerken ist, daß viele Fälle mir schon mit der Wahrscheinlichkeitsdiagnose Ulcus duodeni nur zur Röntgenuntersuchung zugeschickt wurden. Neben vielen Bestätigungen der klinischen Diagnose war die Röntgenuntersuchung aber recht häufig in der Lage, ein Ulcus auszuschließen. Ich halte dies für wichtig gegenüber der an vielen Stellen hervortretenden Neigung, auf ganz vage Symptome hin ein Ulcus zu diagnostizieren. Zum Teil hat hieran unser verdienstvollster Forscher Moynihan selbst schuld, der den Satz aufstellte: „Rezidivierende schwere Hyperazidität ist Ulcus duodeni." Dies trifft für viele Fälle nicht zu.

Daß von 114 nur 23 Fälle operiert sind, hat seinen Grund zum Teil darin, daß wir Internen viel Frühfälle zur Behandlung bekommen, deren Beschwerden zunächst einmal durch eine Ulcuskur beseitigt werden. Ferner aber liegt es in der Natur der Sache, daß wir bestrebt sind, die Eeistungsfähigkeit der inneren Medizin auf ein möglichst hohes Maß zu bringen. Anderseits muß aber davor gewarnt werden, den Termin der Operation zu weit hinauszuschieben; das Nähere hierüber an anderer Stelle.

Was die Schwrerigkeit der Diagnose ganz im allgemeinen betrifft, muß ich mich für die Frühfälle, die chirurgisch noch nicht ausgereift sind, unbedingt auf die Seite derer stellen, welche die Diagnose des Ulcus duodeni für keine leichte halten.

Zusammenfassung. Nur in einem Bruchteil der Fälle weist das Ulcus duodeni im Röntgenbild deutlich erkennbare Veränderungen am Duodenum. selbst auf. Bei der Mehrzahl der Fälle werden uns Indizien für das Vorliegen eines Ulcus dadurch an die Hand gegeben, da $B$ dieses einen intensiven Einfluß auf die Magenfunktionen ausübt. Diese Reflexwirkung des Ulcus auf den Magen ist nicht, wie im allgemeinen angenommen wird, wechselnd, sondern durchaus einheitlich, stets im Sinne einer Funktionssteigerung sich vollziehend. Die dieser Auffassung entgegenstehenden Angaben einiger Autoren beruhen überwiegend darauf, daß Ermüdungs- und Erschöpfungszustände des Magens nicht als solche erkannt wurden. Man bezeichnet den Zustand der reflektorischen Uebererregbarkeit, in dem der Magen sich beim Ulcus duodeni befindet, am besten als reflektorische Exzitationsneurose, die neben einem sonst intakten Nervensystem bestehen kann. Nach längerem Bestehen der funktionellen Uebererregbarkeit stellen sich anatomische Sekundärerscheinungen am Magen ein. Für die Differentialdiagnose ist es wichtig, die primär-funktionellen Aeußerungen dieser Exzitationsneurose und die sekundär-anatomischen Veränderungen auseinanderzuhalten. Primär findet sich: Hyperperistaltik, Hypertonie, von besonderer Bedeutung Hypertonie und Spasmen am Pylorus, ferner Hypersekretion; sekundär: Dauerbulbus, Propulsion des Pylorus nach rechts oben während der peristaltischen Tätigkeit, Dilatation der Pars pylorica und Ausladung derselben nach rechts bis über die Pars verticalis duodeni hinweg, Ektasie des ganzen Magens. Diese anatomischen Veränderungen erst geben der Magenneurose beim Ulcus duodeni ihr besonderes Gepräge und lassen sie von den meisten anderen Neurosen, besonders denen bei Neurasthenie, Cholelithiasis, Appendicitis, Nephrolithiasis u. a., bei denen die Sekundärveränderungen in der Regel fehlen, unterscheiden. Es bleibt aber die Schwierigkeit bestehen, daß die Unterschiede, wenn auch meist markant, so doch nur graduell sind und ihre Differenzierung sehr sorgfältige klinische Erwägungen erfordert. Die praktische Erfahrung hat gelehrt, daß diese in einem hohen Prozentsatz der Fälle von Erfolg begleitet sein können.

Lit e ratur: 1. W. kl. W. 1912 Nr. 11. - 2. M. K1. 1912 Nr. 5 u. 6. - 3. B. kl. W. 1913 Nr. $28 .-4$. D. m. W. 1913 Nr. 51. - 5. M. m. W. 1911 Nr. 23 - 6. Ergebnisse won Magenutersuchugen Won Magenuntersuchungen mittels Rontgenstrahlen im Săuglings- und spateren Kindes alter. Berlin 1911. - 7. Mitt. Grenzgeb. 26. 1913. H. 3. - 8. Arch. of the Roentgen Ray 1910 Nr. $123 .-9$. W. k1. W. 1912 Nr. 11. - 10. Die Ursachen des chronischen Magent-
geschwürs. - 11. Mitt. Grenzgeb. 25. H. 3 u. 4. - 12. M. m. W. 1913 Nr. 4. - 13. D. m. W. (Beilt. 2. M. K1. H. 7 a. 8) 1913 . 